\title{
Transition from Mixed-Valence to Trivalent Cerium State in $\mathrm{Ce}(\mathrm{Ni}, \mathrm{Cu}) \mathrm{Al}$ Series
}

\author{
P. Č́ERMÁK ${ }^{a, *}$, P. JAVORSKÝ ${ }^{a}$ AND E. ŠAnTAVÁ ${ }^{b}$ \\ ${ }^{a}$ Charles University in Prague, Faculty of Mathematics and Physics, Department of Condensed Matter Physics \\ Ke Karlovu 5, 12116 Prague 2, Czech Republic \\ ${ }^{b}$ Institute of Physics AS CR, v. v. i., Na Slovance 2, 18221 Prague 8, Czech Republic
}

\begin{abstract}
In this work we present the study of the part of $\mathrm{Ce}(\mathrm{Ni}, \mathrm{Cu}) \mathrm{Al}$ series from the pure $\mathrm{CeNiAl}$ to $30 \%$ of copper concentration, which illustrates the transition from mixed-valence state of CeNiAl to the trivalent state in CeCuAl. The work is based on X-ray diffraction, magnetization and specific heat measurement. The results indicate smooth transition between the mixed-valence and trivalent cerium state. The specific-heat data reveal increase of the Sommerfeld $\gamma$ coefficient with copper concentration.
\end{abstract}

PACS numbers: 75.40.Cx, 75.30.Mb

\section{Introduction}

The cerium-based compounds show varying ground states as a result of the hybridization between the conduction and the $4 f$-electron states. It is known that the cerium valence can change from trivalent to tetravalent [1]. Special case between these two states is called mixed-valence/intermediate-valence behavior. Broad shallow maximum on the inverse susceptibility curve is one of the typical indications of this state.

Both $\mathrm{CeNiAl}$ and $\mathrm{CeCuAl}$ compounds crystallize in the hexagonal ZrNiAl-type structure (space group $P-62 m$ ). Previous studies on CeNiAl [2,3] showed the attributes of a non-magnetic mixed-valence system with a low $\gamma$-value $\left(\approx 12 \mathrm{~mJ} /\left(\mathrm{mol} \mathrm{K}^{-2}\right)\right)$ of the electronic specific heat. The magnetic susceptibility does not show the Curie-Weiss behavior; instead shows shallow minimum around $150 \mathrm{~K}$ and then it weakly increases with increasing temperature up to $350 \mathrm{~K}$. CeNiAl shows no indication of magnetic ordering down to $2 \mathrm{~K}$. On the other hand, $\mathrm{CeCuAl}$ has clear trivalent state $\mathrm{Ce}^{3+}$, it orders magnetically at temperatures below $5 \mathrm{~K}$ and the specific heat indicate enhanced $\gamma$-value for $\mathrm{CeCuAl}[2]$.

\section{Experimental}

The polycrystalline samples of $\mathrm{CeNi}_{1-x} \mathrm{Cu}_{x} \mathrm{Al}$ with $x=0,0.05,0.1,0.2$ and 0.3 were prepared by melting stoichiometric mixtures of the pure elements in a mono-arc furnace under protection of an argon atmosphere. Every sample was turned and re-melted several times to achieve

* corresponding author; e-mail: cermak@mag.mff.cuni.cz better homogeneity. The X-ray analysis revealed presence of impurities in the copper containing samples. The amount of impurity phases was relatively small $(<5 \%)$ for $x$ up to 0.3 , whereas samples with higher content of $\mathrm{Cu}$ contained larger amount of impurities and were not used for further measurements. The previous studies showed that the synthesis of $\mathrm{CeCuAl}$ is rather difficult, while it does not melt congruently. An annealing treatment enhances sample quality, but never eliminates all impurities phases [4].

The magnetization was measured on powder samples with grains randomly oriented and fixed by glue. Measurements were performed on MPMS (Quantum Design) instrument in a temperature range from 1.8 to $300 \mathrm{~K}$ and in external magnetic fields up to $7 \mathrm{~T}$. The specific heat was measured using a PPMS instrument at temperatures between 0.35 and $300 \mathrm{~K}$ and in magnetic fields up to $3 \mathrm{~T}$. Small samples with a mass of about $2 \mathrm{mg}$ were measured at temperatures below $5 \mathrm{~K}$ whereas larger samples $(\approx 20 \mathrm{mg}$ ) were used for measurements up to $300 \mathrm{~K}$, in order to achieve reasonable precision at higher temperatures.

\section{Results and discussion}

The X-ray diffraction confirmed the hexagonal ZrNiAl-type of structure. The lattice parameters $a$ and $c$ show linear increase with $x: a=697.0(1), 698.4(2), 699.9(1)$, $702.3(2)$ and $705.8(1) \mathrm{pm}, \quad c=402.1(1), 403.2(1)$, $403.8(1), 405.5(1)$ and 407.1(1) pm for $x=0.0,0.05$, $0.10,0.20$ and 0.30 , respectively. These values are in agreement with the values for pure $\mathrm{CeNiAl}$ and $\mathrm{CeCuAl}$ $[2,3,5]$.

The $M / H$ vs. $T$ dependences for all samples are shown in Fig. 1 together with previously measured data on pure 
$\mathrm{CeCuAl}[2]$. The effective moment per one $\mathrm{Ce}$ atom $\mu_{\text {eff }}=2.18 \mu_{\mathrm{B}}$ is reported for the pure $\mathrm{CeCuAl}$ [2]. It is lower than the $\mathrm{Ce}^{3+}$ free-ion value of $2.54 \mu_{\mathrm{B}}$. The lower value was ascribed to nonmagnetic impurities [2]. Determination of $\mu_{\text {eff }}$ as well as the paramagnetic Curie temperature $\theta_{p}$ is not possible for our samples, because none of them exhibits the Curie-Weiss behavior, indicating mixed-valence state. We can observe gradual transition from the mixed-valence to the Curie-Weiss behavior, indicated by disappearance of maximum in the $H / M(T)$ curve. Magnetization curves are shown in Fig. 2. They do not exhibit any hysteresis. Very small measured magnetic moments are consistent with the mixed-valence behavior. We can observe increase of magnetic moments with increasing copper concentration.

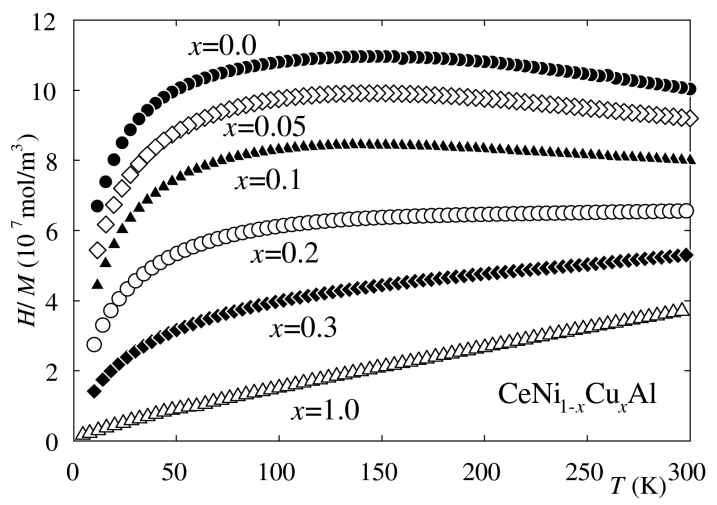

Fig. 1. $H / M(T)$ dependences measured in field of $2 \mathrm{~T}$.

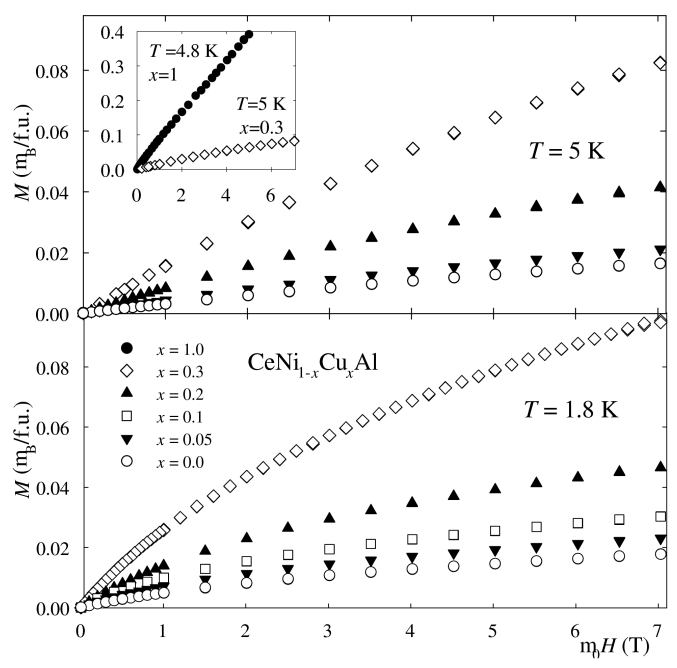

Fig. 2. Magnetization curves measured at 1.8 and $5 \mathrm{~K}$. The inset represents comparison of $\mathrm{CeNi}_{0.7} \mathrm{Cu}_{0.3} \mathrm{Al}$ and $\mathrm{CeCuAl}[2]$.

All measured $C_{p}(T)$ dependences are shown in Fig. 3 . In the low temperature region we observe a small anomaly around $6 \mathrm{~K}$, which can be ascribed to cerium oxide contamination (phase transition at $6.2 \mathrm{~K}$ ). Cer- tain anomaly seems to be present also around $2 \mathrm{~K}$. This anomaly becomes larger and shifts to $2.5 \mathrm{~K}$ for $x=0.3$. However, entropy of this anomaly is still very small $(\approx 5 \%$ of $R \ln 2$ ) compared to entropy expected for a magnetic order of a localized system with doublet ground state. Although the magnetic entropy of cerium systems is often reduced due to e.g. the Kondo effect, the observed entropy is much smaller also compared to other Ce compounds including $\mathrm{CeCuAl}$ where the magnetic entropy reaches $\approx 0.4 R \ln 2[2]$. Considering also the magnetization data, we assume that $\mathrm{CeNi}_{0.7} \mathrm{Cu}_{0.3} \mathrm{Al}$ still cannot be treated as a $3+$ system and the observed anomaly is only an indication of a weak magnetic order.

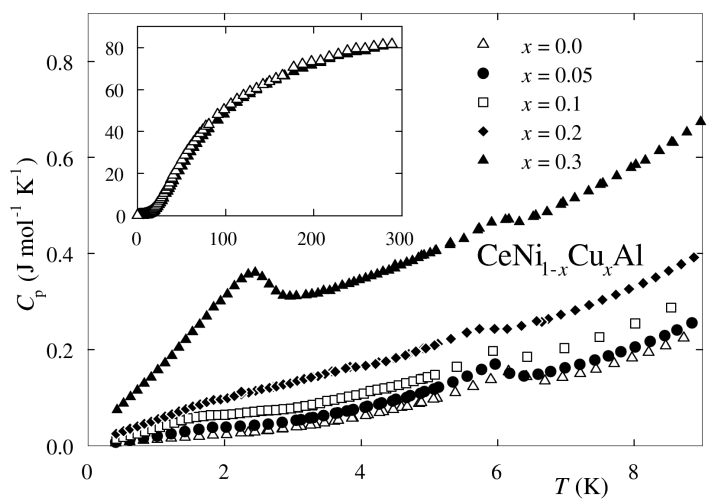

Fig. 3. The low-temperature specific heat of the $\mathrm{CeNi}_{1-x} \mathrm{Cu}_{x} \mathrm{Al}$ compounds. The inset shows whole temperature comparison of compounds with $x=0.0$ and 0.3 .

The quantitative analysis of the $C_{p}(T)$ dependence in the whole temperature range up to $300 \mathrm{~K}$ is almost impossible because of missing reliable estimation of the phonon part (the La analogs crystallize in a different structure [6]). Nevertheless, we can make some qualitative conclusions about the electronic specific heat. The $\gamma$ -value clearly increases with copper concentration to values above $100 \mathrm{~mJ} \mathrm{~mol}^{-1} \mathrm{~K}^{-2}$ for $x=0.3$. These values correspond to the low temperature region. Above $50 \mathrm{~K}$, the data for different compounds are almost the same (see inset in Fig. 3) which means that, assuming similar phonon part, the high temperature $\gamma$-values are similar for all concentrations being around $10 \mathrm{~mJ} \mathrm{~mol} \mathrm{~K}^{-1} \mathrm{~K}^{-2}$ as reported for $\mathrm{CeNiAl}$ [2]. The large difference between the high and low-temperature $\gamma$-value for $x>0.1$ implies temperature dependent $\gamma$-value as in analogous $\mathrm{CePdAl}$ compound [7].

\section{Conclusions}

We succeeded in preparation of the compounds from the $\mathrm{Ce}(\mathrm{Ni}, \mathrm{Cu}) \mathrm{Al}$ series up to $30 \%$ of copper concentration. The magnetization and specific heat data showed gradual transition from the mixed-valence state in $\mathrm{CeNiAl}$ to the $\mathrm{Ce}^{3+}$ state in $\mathrm{CeCuAl}$. This development is accompanied by an increase of low temperature $\gamma$ with increasing copper content. 


\section{Acknowledgments}

This work is a part of the research program MSM 0021620834 financed by the Ministry of Education of the Czech Republic. The work of P.C. was supported by the grant SVV-2010-261303.

\section{References}

[1] A.S. Edelstein, J. Magn. Magn. Mater. 256, 430 (2003).

[2] P. Javorský, A. Chernyavsky, V. Sechovský, Physica B 281-282, 71 (2000).
[3] N.C. Tuan, Ph.D. Thesis, Prague 1992.

[4] B. Chevalier, J. Bobet, Intermetallics 9, 835 (2001).

[5] H. Oesterreicher, J. Less-Common Met. 30, 225 (1973).

[6] G. Cordier, G. Dörsam, R. Kniep, J. Magn. Magn. Mater. 76-77, 653 (1988).

[7] P. Čermák, H. Kitazawa, J. Prchal, P. Javorský, J. Phys., Condens. Matter 22, 126002 (2010). 